\title{
Role of enteroviruses in the pathogenesis of type 1 diabetes
}

\author{
M. Roivainen $\cdot$ K. Klingel
}

Received: 17 February 2009 / Accepted: 18 February 2009/Published online: 26 March 2009

(C) Springer-Verlag 2009

Keywords Beta cells · Enterovirus · Enterovirus detection · Enterovirus infection $\cdot$ Type 1 diabetes $\cdot$ Vp1 immunostaining

The pathogenesis of type 1 diabetes is widely believed to involve $\mathrm{T}$ cell-mediated autoimmune processes directed against the insulin-producing beta cells in the pancreas. Growing evidence suggests that environmental factors, including toxins, food antigens and, particularly, viral infections are involved in the induction of type 1 diabetes. Viruses such as enteroviruses, rubella virus, mumps virus, rotavirus, parvovirus and cytomegalovirus have been investigated in experimental and clinical studies aimed at defining their roles in the pathogenesis of type 1 diabetes. Most efforts in recent years have focused on enteroviruses, the most common cause of viral infection in humans, infecting an estimated billion people annually worldwide [1]. Enteroviruses exhibit islet cell tropism, as demonstrated by the detection of viral RNA by in situ hybridisation and the identification of viral proteins by immunohistochemical staining of post-mortem pancreatic specimens from type 1 diabetogenic patients [2, 3]. Furthermore, an infectious coxsackievirus B4 was isolated from the islets of a type 1 diabetic patient [2], and a strain of echovirus 3 was isolated

\footnotetext{
M. Roivainen ( $\square)$

National Institute for Health and Welfare (THL),

P.O. Box 30, 00271 Helsinki, Finland

e-mail: merja.roivainen@thl.fi

K. Klingel

Department of Molecular Pathology,

University Hospital of Tübingen,

Tübingen, Germany
}

from an individual concurrently with appearance of islet cell and IA-2 autoantibodies [4].

The study by Richardson and collaborators [5], published in this issue of Diabetologia, is a welcome addition to the field, supporting the relevance of enteroviruses as diabetes-inducing agents. The authors have studied highly valuable human material-the autopsy pancreases of recent-onset diabetic patients - for the presence of enteroviruses. Enteroviral antigen-positive islet cells were found in 44 of 72 young recent-onset type 1 diabetic patients, in line with the original findings of other groups [2, 3]. Furthermore, islets from ten of 25 cases with type 2 diabetes tested positive for the enteroviral capsid protein vp1 by immunohistochemistry [5], pointing to a possible role of these viruses in the aetiopathogenesis of this disease as well. As negative controls, they investigated 50 pancreases from neonatal or paediatric non-diabetic individuals, reporting three vp1-positive samples. In the studied islet preparations, vp1 staining was restricted to beta cells and correlated well with the immunostaining of double-stranded RNA-activated protein kinase $\mathrm{R}$. With the help of this enzyme, the authors aimed to confirm that the vp1-positive insulin-containing cells represented virus-infected cells.

Human enteroviruses comprise a large group of pathogens that are subdivided into four species - A, B, C and Dwith more than 100 different serotypes. All the enterovirus serotypes investigated to date, in prospective studies, islet cell culture or animal studies, appear to include strains with the ability to damage beta cells. In contrast to previous studies that used serotype-specific methods, recent progress in the field of enterovirus-associated type 1 diabetes has, to a large extent, involved the application of enterovirus group-specific verification procedures. There is an antibody on the market (clone 5D8/1; Dako, Glostrup, Denmark) that 
seems suitable for the detection of $\mathrm{vp} 1$ in paraffinembedded tissues. Thus, the majority of studies that have used immunohistochemical staining for the detection of enteroviruses, including the study by Richardson et al., have employed this monoclonal antibody $[2,5,6]$. These studies rely on an immunohistochemical staining method that shows high sensitivity for the detection of enteroviruses, since this antibody is targeted to the highly conserved immunodominant region of the capsid protein and can therefore react with a wide range of different enterovirus serotypes. High sensitivity of an antibody may be beneficial in a preliminary search for virus infections, but it may also bring problems in terms of non-specific reactions. The observation of positive staining of focal pancreatic centoacinar cells and smooth muscle cells by the Dako anti-vp1 serum in some adults described by Richardson et al. [5] indicates that there is nonspecific staining by this antibody in pancreatic tissues, because enteroviruses have never been shown to be capable of infecting these types of cells. The finding of occasional non-specific binding to cellular proteins by this Dako antibody is supported by immunohistochemical staining of hearts from patients with dilated cardiomyopathy, illustrating a considerable cross-reaction of this antibody with cellular proteins in uninfected human cardiomyocytes [7]. It should be noted that enterovirus signals in islets of diabetic patients may involve only a few cells, whereas the signals in islets of neonates who died from fulminant coxsackievirus infections may be much more impressive [3]. Therefore, potential positive immunohistochemical findings should be confirmed by other methods, i.e. by isolating an infectious virus or demonstrating the presence of virus genomes by RT-PCR or by radioactive in situ hybridisation. The application of this highly specific in situ hybridisation assay is facilitated by the fact that enterovirus RNA is profoundly stable, and Ylipaasto et al. [3] demonstrated that it is detectable in paraffin-embedded pancreatic tissue samples older than 30 years. As no enterovirus protein is translated without viral RNA, it is likely that positive immunostaining for vp1 in the absence of viral RNA indicates recognition of cellular antigens by the antibody. In fact, the highly conserved Nterminal immunodominant region of vp1 shares sequence similarity with the known diabetes-associated epitopes of two islet cell autoantigens, the tyrosine phosphatases (IA-2/IAR) and heat shock protein 60/65 [8,9]. This also explains why the inclusion of other virus capsid-recognising antibodies in this paper, e.g. the polyclonal antibody against vp1 of coxsackievirus B3 [10], may not be conclusive proof of the presence of enterovirus.
The paper by Richardson et al. [5] may reinforce the potential relevance of enteroviruses in the induction of diabetes. However, given the potential importance of this finding for the field, further studies using other surrogate markers of viral infection are needed to corroborate these results and to establish a causative relationship between enterovirus infection and the development of type 1 diabetes.

Duality of interest The authors declare that there is no duality of interest associated with this manuscript.

\section{References}

1. Oberste MS, Pallansch MA (2003) Establishing evidence for enterovirus infection in chronic disease. Ann N Y Acad Sci 1005:23-31

2. Dotta F, Censini S, van Halteren AG et al (2007) Coxsackie B4 virus infection of beta cells and natural killer cell insulitis in recent-onset type 1 diabetic patients. Proc Natl Acad Sci USA 104:5115-5120

3. Ylipaasto P, Klingel K, Lindberg AM et al (2004) Enterovirus infection in human pancreatic islet cells, islet tropism in vivo and receptor involvement in cultured islet beta cells. Diabetologia 47:225-239

4. Williams CH, Oikarinen S, Tauriainen S, Salminen K, Hyoty H, Stanway G (2006) Molecular analysis of an echovirus 3 strain isolated from an individual concurrently with appearance of islet cell and IA-2 autoantibodies. J Clin Microbiol 44:441-448

5. Richardson SJ, Willcox A, Bone AJ, Foulis AK, Morgan NG (2009) The prevalence of enteroviral capsid protein vp1 immunostaining in pancreatic islets in human type 1 diabetes. Diabetologia. doi:10.1007/s00125-009-1276-0

6. Oikarinen M, Tauriainen S, Honkanen T et al (2008) Analysis of pancreas tissue in a child positive for islet cell antibodies. Diabetologia 51:1796-1802

7. Klingel K, Sauter M, Bock CT, Szalay G, Schnorr JJ, Kandolf R (2004) Molecular pathology of inflammatory cardiomyopathy. Med Microbiol Immunol 193:101-107

8. Harkonen T, Lankinen H, Davydova B, Hovi T, Roivainen M (2002) Enterovirus infection can induce immune responses that cross-react with $\beta$-cell autoantigen tyrosine phosphatase IA-2/IAR. J Med Virol 66:340-350

9. Harkonen T, Puolakkainen M, Sarvas M, Airaksinen U, Hovi T, Roivainen M (2000) Picornavirus proteins share antigenic determinants with heat shock proteins 60/65. J Med Virol 62:383-391

10. Werner S, Klump WM, Schonke H, Hofschneider PH, Kandolf R (1988) Expression of coxsackievirus B3 capsid proteins in Escherichia coli and generation of virus-specific antisera. DNA 7:307-316 